\title{
Ablation of silicon suboxide thin layers
}

\author{
M. Jahn - J. Richter $\cdot$ R. Weichenhain-Schriever . \\ J. Meinertz $\cdot$ J. Ihlemann
}

Received: 17 November 2009 / Accepted: 24 May 2010 / Published online: 3 July 2010

(C) Springer-Verlag 2010

\begin{abstract}
We investigate the ablation of $\mathrm{SiO}_{x}$ thin films on fused silica substrates using single-pulse exposures at $193 \mathrm{~nm}$ and $248 \mathrm{~nm}$. Two ablation modes are considered: front side (the surface of a film is irradiated from above) and rear side (a film is irradiated through its supporting substrate). Fluence is varied from below $200 \mathrm{~mJ} / \mathrm{cm}^{2}$ to above $3 \mathrm{~J} / \mathrm{cm}^{2} . \mathrm{SiO}_{x}$ films of thickness $200 \mathrm{~nm}, 400 \mathrm{~nm}$, and $600 \mathrm{~nm}$ are ablated. In the case of rear-side illumination, at moderate fluences (around $0.5 \mathrm{~mJ} / \mathrm{cm}^{2}$ ) the ablation depth corresponds roughly to the film thickness, above $1 \mathrm{~J} / \mathrm{cm}^{2}$ part of the substrate is ablated as well. In the case of frontside ablation the single-pulse ablation depth is limited for all film thicknesses to less than $200 \mathrm{~nm}$ even at fluences up to $4 \mathrm{~J} / \mathrm{cm}^{2}$. Experimental results are discussed in relation to film thickness, fluence, and ablation mode. Simple numerical calculations are performed to clarify the influence of heat transport on the ablation process.
\end{abstract}

\section{Introduction}

Because of its high transparency throughout the UV/VIS/ NIR spectral region fused silica $\left(\mathrm{SiO}_{2}\right)$ finds widespread use in optical components. Excimer-grade fused silica has sufficiently high transmission to permit its use in laser optics even at $193 \mathrm{~nm}$ (ArF excimer laser). The small absorption in the DUV, however, makes processing of fused silica by excimer laser ablation rather difficult. While ablation at $193 \mathrm{~nm}$ is possible at high fluences (in excess of $2 \mathrm{~J} / \mathrm{cm}^{2}$ )

M. Jahn · J. Richter $\cdot$ R. Weichenhain-Schriever .

J. Meinertz $(\bowtie) \cdot$ J. Ihlemann

Laser-Laboratorium Göttingen e. V., Hans-Adolf-Krebs-Weg 1,

37077 Göttingen, Germany

e-mail: joerg.meinertz@llg-ev.de the ablation rate is found to depend on the surface roughness and irradiation by multiple pulses can lead to crack formation [1]. Processing of fused silica at $157 \mathrm{~nm}\left(\mathrm{~F}_{2}\right.$ laser) has been performed by a number of groups and interesting applications have been demonstrated [2-5]. The experimental effort, however, is very high: beam lines have to be evacuated or purged to remove any absorbing oxygen and laser optics have to be all-reflective or made from expensive materials like calcium fluoride. Also, the available pulse energy is normally less for $\mathrm{F}_{2}$ lasers.

To circumvent these problems indirect methods have been invented that permit processing of fused silica at standard excimer laser wavelengths. These methods employ an auxiliary medium which absorbs the UV laser photons and transfers some of the energy to the fused silica resulting in material removal. Some of these methods and variations thereof are known as laser-induced backside wet-etching (LIBWE) [6-8], laser-induced plasma assisted ablation (LIPAA) [9], laser etching at a surface adsorbed layer (LESAL) [10], and, more recently, laserinduced backside dry etching (LIBDE) [11]. LIBDE, which commonly uses metal absorbers, has been thoroughly studied $[12,13]$ and is a promising technique for laser microprocessing of transparent materials. In recent years we have developed an alternative method for excimer laser micromachining of fused silica [14]. As absorbing medium we use non-stoichiometric silicon oxide $\left(\mathrm{SiO}_{x}\right)$ thin films coated on fused silica substrates. $\mathrm{SiO}_{x}$ has sufficiently high absorption to permit ablation at $248 \mathrm{~nm}(\mathrm{KrF}$ excimer laser) and $193 \mathrm{~nm}$. After ablation the remaining $\mathrm{SiO}_{x}$ film can be oxidized to $\mathrm{SiO}_{2}$, e.g. by baking in an oven, yielding a UVtransparent component completely composed of $\mathrm{SiO}_{2}$ [15]. Baking takes around 8 hours for $\mathrm{SiO}_{x}$ films that are $200 \mathrm{~nm}$ thick. A $600 \mathrm{~nm} \mathrm{SiO}_{x}$ film requires up to 24 hours of baking for complete oxidation so the method is better suited for thin films. 
In rear-side ablation (RSA) irradiation is performed through the substrate. The interface between the nonabsorbing fused silica substrate and the absorbing $\mathrm{SiO}_{x}$ film then functions as a predetermined breaking point, so film ablation leaves behind the substrate surface which has a low roughness. The combination of rear-side $\mathrm{SiO}_{x}$ film ablation followed by oxidation to $\mathrm{SiO}_{2}$ can be used to produce high-quality UV-transparent phase masks very costefficiently [16].

Though similar, our method differs from LIBDE in some key points. Firstly, laser processing is normally performed below the threshold for indirect ablation, i.e. the transparent substrate is not etched. And secondly, the remaining $\mathrm{SiO}_{x}$ film is left on the substrate and converted to $\mathrm{SiO}_{2}$ by oxidation becoming an intrinsic part of the surface pattern. In LIBDE the film is removed by some wet-chemical agent. Therefore, etch depths for LIBDE directly refer to the transparent substrate. In our method, they refer to the film-onsubstrate system. This is to be kept in mind when comparing both methods. In the following, results on front-side ablation (FSA) and RSA of $\mathrm{SiO}_{x}$ thin films are presented.

\section{Experiment}

Ablation experiments on silicon suboxide $\left(\mathrm{SiO}_{x}, x<2\right)$ thin films were performed at $193 \mathrm{~nm}$ using a Lambda Physik LPX 300 ArF excimer laser and at $248 \mathrm{~nm}$ using a Lambda Physik EMG 300 MSC KrF excimer laser. Samples consisted of excimer-grade (UV-transparent) fused silica substrates coated with single layers of $\mathrm{SiO}_{x}$ of a prescribed thickness. For the samples discussed in this paper, the nominal film thickness ranges from $20 \mathrm{~nm}$ to $600 \mathrm{~nm}$. Coating was performed by a commercial coating shop (Laseroptik, Garbsen, Germany). Although this coating shop labels the films as silicon monoxide $(x=1)$, the oxygen content $x$ is not known exactly and may vary between samples belonging to different batches. However, all investigated films show strong absorption in the UV. Transmission measurements with a UV/VIS/NIR spectrometer (Perkin Elmer Lambda 19) on a $84 \mathrm{~nm} \mathrm{SiO}_{x}$ film provided estimates of the absorption coefficient $\alpha$ of $4.6 \times 10^{5} \mathrm{~cm}^{-1}$ at $193 \mathrm{~nm}$ and $2.7 \times 10^{5} \mathrm{~cm}^{-1}$ at $248 \mathrm{~nm}$. Film transmission in the VIS region is around 0.8 to 0.9 and shows Fabry-Pérot resonances originating from multiple reflections in the film. From these resonances and the known film thicknesses a crude (wavelength-independent) estimate of the refractive index of around 1.9 can be obtained. This is in good agreement with refractive index data for $\mathrm{SiO}$ graphed in [17] for the wavelength region from $240 \mathrm{~nm}$ to $1000 \mathrm{~nm}$. Finally, film thickness increases by a factor of approximately 1.2 to 1.4 upon oxidation and UV-transmission rises to values characteristic of fused silica. This points to a significant incorpo- ration of oxygen so it is reasonable to assume $1 \approx x \ll 2$ for the $\mathrm{SiO}_{x}$ films.

The ablation experiments were carried out using a maskprojection setup. Employed masks included simple geometries like circular apertures and slits as well as more complex ones like gratings and concentric rings. Depending on mask and demagnification ratio the obtained feature size of ablated structures ranged from a few to several hundred microns. Laser fluence was controlled with a variable attenuator, the energy of the laser pulses being measured using a standard joulemeter. FSA and RSA modes were investigated. In FSA mode the sample is mounted with the $\mathrm{SiO}_{x}$ film facing the incident laser beam while in RSA mode the laser pulse first travels through the fused silica substrate before being absorbed by the film. In this latter mode care has to be taken because the substrate leads to an axial shift of the projected mask image. This has been accounted for in the experiments. All ablations were carried out at room temperature and atmospheric pressure.

\section{Results and discussion}

Figure 1 shows confocal microscope images of cavities ablated in a $400 \mathrm{~nm} \mathrm{SiO}_{x}$ film using FSA and RSA at $193 \mathrm{~nm}$. For these results a chromium on calcium fluoride mask with a square aperture was imaged by a spherical lens onto the film at a demagnification of 10:1. The size of the aperture, $1 \times 1 \mathrm{~mm}^{2}$, was chosen so that inhomogeneities of the laser beam would have a negligible effect on the ablation results. Fluence was varied from around $200 \mathrm{~mJ} / \mathrm{cm}^{2}$ to above $3 \mathrm{~J} / \mathrm{cm}^{2}$. Around $350 \mathrm{~mJ} / \mathrm{cm}^{2}$ FSA (Fig. 1a) and RSA (Fig. 1b) produce cavities with well-defined edges while around $3 \mathrm{~J} / \mathrm{cm}^{2}$ FSA (Fig. 1c) and RSA (Fig. 1d) result in cavities with blurred edges. An obvious difference between Fig. 1c and Fig. 1d is the roughness of the cavity bottoms. The roughness resulting from FSA (Fig. 1c) is higher than that resulting from RSA (Fig. 1d). This observation holds over the whole investigated fluence range, irrespective whether just the film is ablated or part of the substrate, tooFSA produces rougher cavity bottoms than RSA. In fact, the confocal microscope data for FSA are so noisy that we have not been able to reliably quantify ablation depths while this would have been possible for most of the measurements on cavities produced by RSA. For this reason etch depths were determined independently with a Sloan Dektak 3030 stage profiler.

Figure 2 shows the obtained single-pulse etch depths for

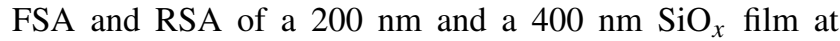
$193 \mathrm{~nm}$. Also shown are data for FSA of a $200 \mathrm{~nm}$ and a $600 \mathrm{~nm} \mathrm{SiO} x$ film at $248 \mathrm{~nm}$. From these data one can see that the ablation threshold for FSA is lower at $193 \mathrm{~nm}$ than at $248 \mathrm{~nm}$. This is expected because of the stronger absorption of $\mathrm{SiO}_{x}$ at $193 \mathrm{~nm}$. At $248 \mathrm{~nm}$ there is no ablation below 

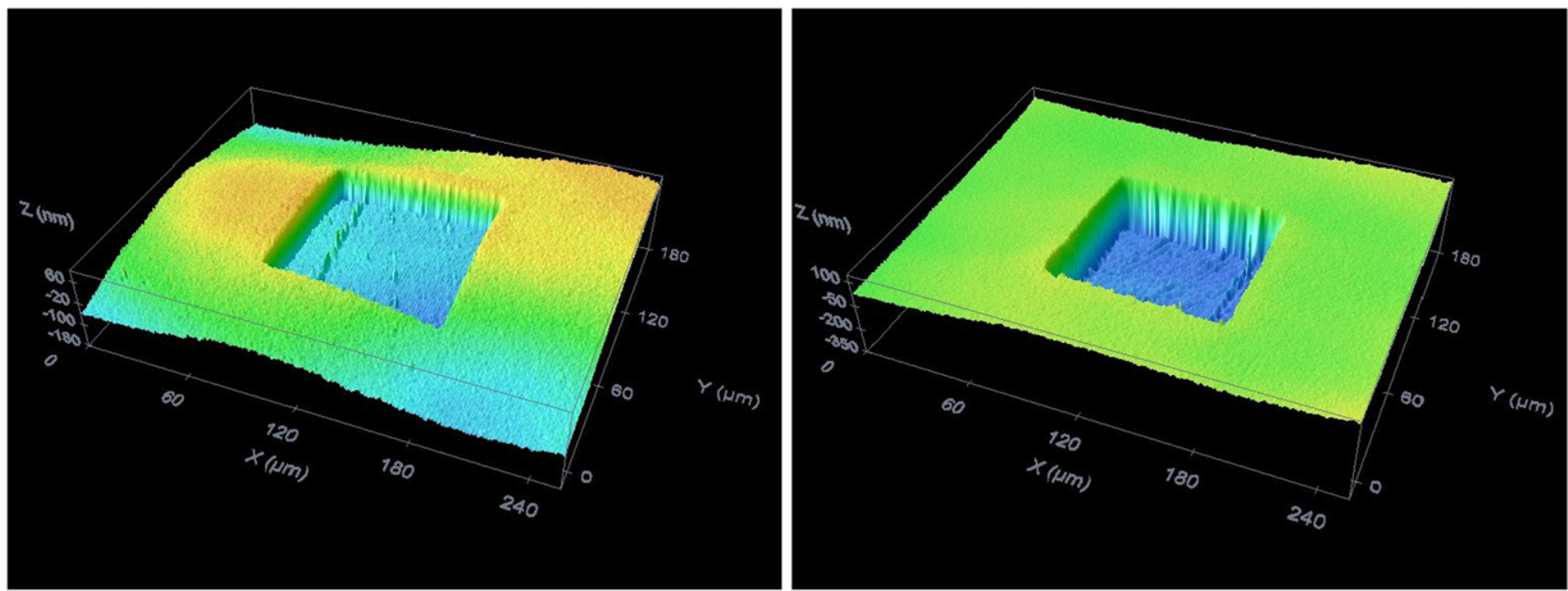

a $\mathbf{b}$
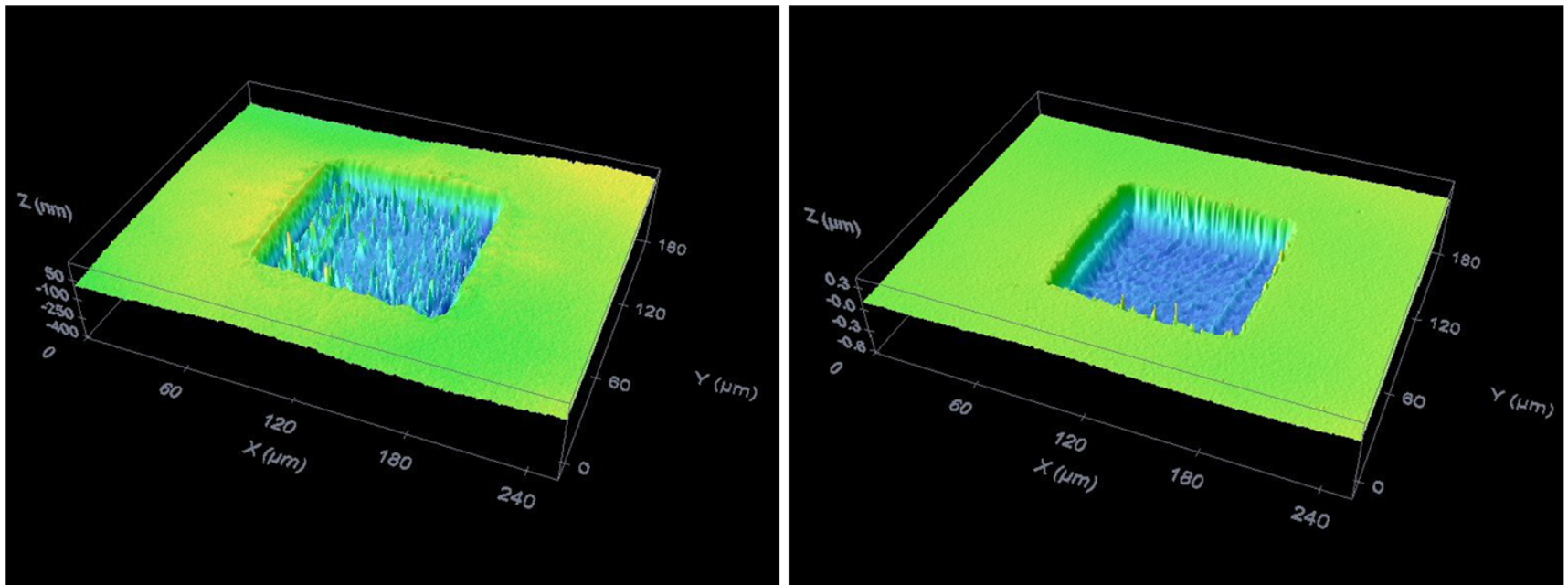

c

d

Fig. 1 Single-pulse ablation of a $400 \mathrm{~nm}$ thick $\mathrm{SiO}_{x}$ film at $193 \mathrm{~nm}$; (a) FSA, $370 \mathrm{~mJ} / \mathrm{cm}^{2}$; (b) RSA, $340 \mathrm{~mJ} / \mathrm{cm}^{2}$; (c) FSA, $3.2 \mathrm{~J} / \mathrm{cm}^{2}$; (d) RSA, $3.0 \mathrm{~J} / \mathrm{cm}^{2}$. Measurements by confocal microscope. Note different $z$ scales

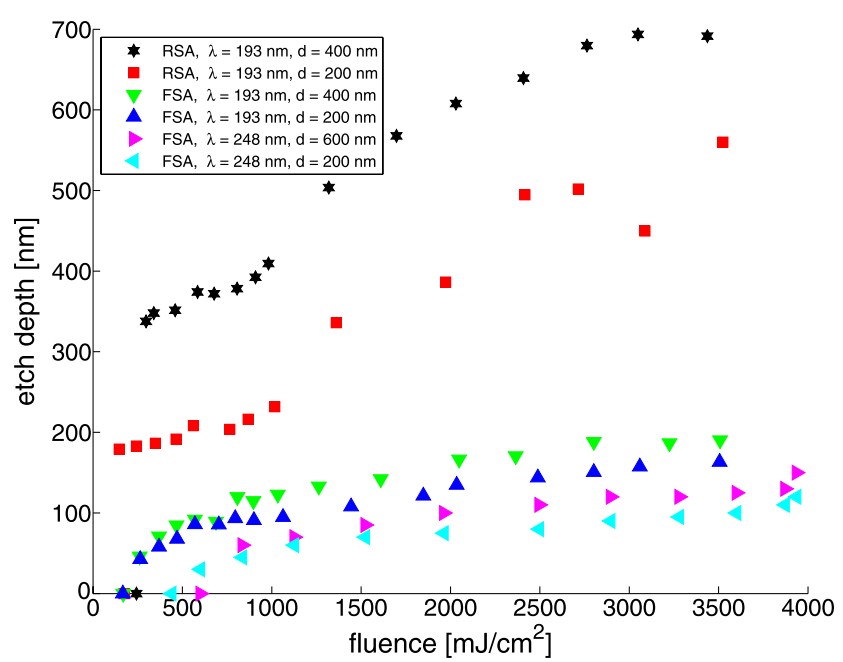

Fig. 2 Single-pulse ablation depth versus fluence for $\mathrm{SiO}_{x}$ films of varying thickness; $\lambda$ : wavelength; $d$ : film thickness
$500 \mathrm{~mJ} / \mathrm{cm}^{2}$. At $193 \mathrm{~nm}$ single-pulse etch depths of $43 \mathrm{~nm}$ (200 nm film) and $47 \mathrm{~nm}$ (400 nm film) are observed around $260 \mathrm{~mJ} / \mathrm{cm}^{2}$ while no ablation is observed at $170 \mathrm{~mJ} / \mathrm{cm}^{2}$ (both films). Between $170 \mathrm{~mJ} / \mathrm{cm}^{2}$ and $260 \mathrm{~mJ} / \mathrm{cm}^{2}$ data obtained from the stage profiler are inconclusive because the irradiated area becomes rougher before ablation sets in. This is supported by the confocal microscope measurements. The etch depth increases with fluence for both wavelengths and all films. However, for FSA, measured etch depths remain below the film thickness in all cases, at least within the fluence range of this study. We believe the main reason for this is plasma shielding of the laser pulse by the ablation plume. The largest etch depths measured for FSA at $193 \mathrm{~nm}$ were $190 \mathrm{~nm}\left(3.5 \mathrm{~J} / \mathrm{cm}^{2}, 400 \mathrm{~nm}\right.$ film $)$ and $163 \mathrm{~nm}\left(3.5 \mathrm{~J} / \mathrm{cm}^{2}\right.$, $200 \mathrm{~nm}$ film). For FSA at $248 \mathrm{~nm}$ the data are $150 \mathrm{~nm}$ $\left(3.9 \mathrm{~J} / \mathrm{cm}^{2}, 600 \mathrm{~nm}\right.$ film) and $120 \mathrm{~nm}\left(3.9 \mathrm{~J} / \mathrm{cm}^{2}, 200 \mathrm{~nm}\right.$ film). One final observation about the data on FSA is that, firstly, for a fixed fluence ablation depths are consistently 
larger at $193 \mathrm{~nm}$ than at $248 \mathrm{~nm}$ and, secondly, that for a fixed fluence and wavelength ablation depths are consistently larger for thicker than for thinner films. The first effect is more pronounced than the second. The reasons for this are still under investigation. With respect to the thickness dependence it has to be noted, however, that because of roughness the measurement error of the ablation depth is of the same order of magnitude as the differences observed in the data. Therefore, this effect is hard to verify experimentally.

For RSA at $193 \mathrm{~nm}$ incomplete film removal is observed at $174 \mathrm{~mJ} / \mathrm{cm}^{2}$ for the $200 \mathrm{~nm} \mathrm{SiO}$ film and at $297 \mathrm{~mJ} / \mathrm{cm}^{2}$ for the $400 \mathrm{~nm}$ film. In some parts of the irradiated area the film is removed, in others it is still on the substrate. Complete film removal is observed at $243 \mathrm{~mJ} / \mathrm{cm}^{2}$ (200 nm film) and at $340 \mathrm{~mJ} / \mathrm{cm}^{2}$ (400 nm film). For completeness we also mention data obtained in our lab for RSA at $248 \mathrm{~nm}$. Complete removal of an $\mathrm{SiO}_{x}$ film $200 \mathrm{~nm}$ thick requires around $400 \mathrm{~mJ} / \mathrm{cm}^{2}$ while around $600 \mathrm{~mJ} / \mathrm{cm}^{2}$ are needed for a $600 \mathrm{~nm}$ film. A $20 \mathrm{~nm} \mathrm{SiO} x$ film can be ablated with around $200 \mathrm{~mJ} / \mathrm{cm}^{2}$. These data strongly suggest that there is a positive correlation between film thickness and threshold fluence required for complete film removal, i.e. thicker films require more fluence in RSA. In the low fluence region where complete film removal begins, measured ablation depths come out about 10 percent lower than the nominal film thickness. This suggests that some $\mathrm{SiO}_{x}$ remains on the substrate or is redeposited there during the ablation. With increasing fluence ablation depths first rise slowly, then, at some fluence the ablation depth exceeds the film thickness. From there, ablation depths rise faster with fluence. In this latter regime the substrate is etched. As can be seen for the $200 \mathrm{~nm}$ film this etching of the fused silica substrate can be very effective. At $3.5 \mathrm{~J} / \mathrm{cm}^{2}$ the measured single-pulse ablation depth is $560 \mathrm{~nm}$ which means that $360 \mathrm{~nm} \mathrm{SiO}_{2}$ have been removed in addition to $200 \mathrm{~nm} \mathrm{SiO}_{x}$. It is worthy to note that one does not depend on an $\mathrm{SiO}_{x}$ layer that thick for this effect. A study conducted in our group has demonstrated ablation rates of several hundred nanometers with single-pulse RSA and an absorbing $\mathrm{SiO}_{x}$ film only $28 \mathrm{~nm}$ thick [18].

Figure 3 shows a binary structure of alternating lines and spaces produced by RSA of a $223 \mathrm{~nm}$ thick $\mathrm{SiO}_{x}$ layer. For this structure a chromium on quartz mask was demagnified four times by a $4 \times / 10-248$ objective (MicroLas, Göttingen, Germany). Ablation was performed at $248 \mathrm{~nm}$ using just a single laser pulse. The average fluence, obtained by dividing the measured pulse energy by the total area of the structure, was $200 \mathrm{~mJ} / \mathrm{cm}^{2}$. At a fill factor of 0.5 this gives a calculated fluence of $400 \mathrm{~mJ} / \mathrm{cm}^{2}$ on the grating lines. The single pulse produced a grating with a period of only $2 \mu \mathrm{m}$ covering an area of $4.7 \mathrm{~mm}^{2}$. Figure 3 shows some of the grating lines. Their edges are well-defined so that the structure is

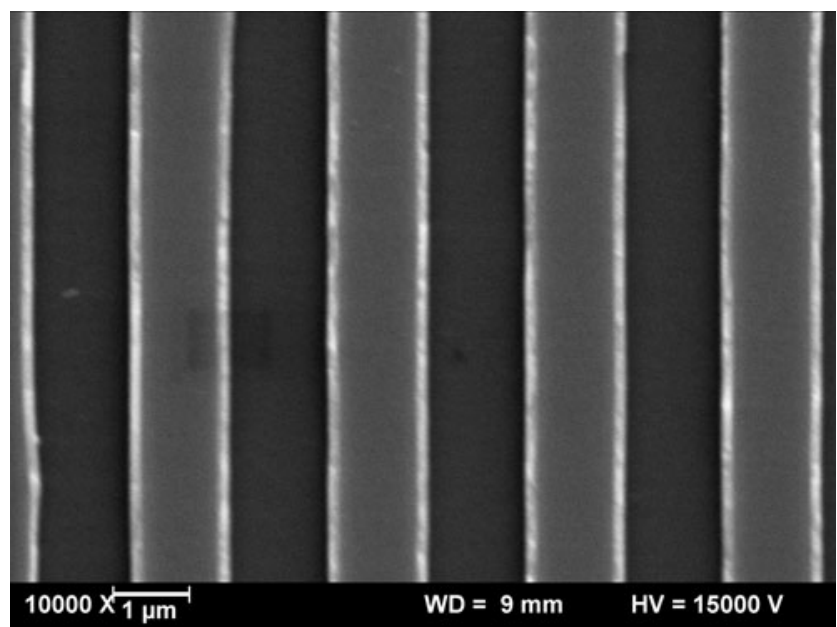

Fig. 3 Binary structure of alternating $1 \mu \mathrm{m}$ wide ridges and trenches produced by single-pulse RSA at $248 \mathrm{~nm}$ of a $223 \mathrm{~nm}$ thick $\mathrm{SiO}_{x}$ layer. $200 \mathrm{~mJ} / \mathrm{cm}^{2}$ average fluence (pulse energy divided by area of grating) corresponding to $400 \mathrm{~mJ} / \mathrm{cm}^{2}$ on the grating lines

indeed binary, i.e. step-function like with two levels defined by the $\mathrm{SiO}_{x}$-air and $\mathrm{SiO}_{x}$-substrate interfaces. This result demonstrates that binary microstructures covering areas of multiple square millimeters and with feature sizes on the micron level can be efficiently produced by single-pulse RSA of $\mathrm{SiO}_{x}$ layers.

We have used a simple thermal model to qualitatively analyze our results. To that end, we have numerically solved the heat-transport equation

$\rho c_{p} \frac{\partial T}{\partial t}=\kappa \frac{\partial^{2} T}{\partial z^{2}}+\alpha I(z, t)$

for a film-on-substrate system irradiated by a laser pulse. Because the irradiated area is large compared to the film thickness, lateral heat conduction is neglected and only the longitudinal coordinate $z$ is kept. $T(z, t)$ and $I(z, t)$ are the temperature and laser intensity, respectively. We treat the density $\rho$, the specific heat capacity $c_{p}$, the thermal conductivity $\kappa$ and the absorption coefficient $\alpha$ as constants (which were allowed to differ between film and substrate). The film surface is taken as an isolating interface which does not permit heat flow and the substrate is modeled as a $2000 \mathrm{~nm}$ thick layer in contact with a heat bath at room temperature. Used parameter values are $\rho=2.13 \mathrm{~g} / \mathrm{cm}^{3}$ [19] for $\mathrm{SiO}_{x}$, $\rho=2.20 \mathrm{~g} / \mathrm{cm}^{3}$ [20] for fused silica, $c_{p}=1.052 \mathrm{~J} /(\mathrm{g} \mathrm{K})$ [20] for fused silica $\kappa=0.015 \mathrm{~W} /(\mathrm{cm} \mathrm{K})$ [20] for fused silica $\alpha=2.7 \times 10^{5} \mathrm{~cm}^{-1}$ for $\mathrm{SiO}_{x}$ at $248 \mathrm{~nm}$ (own estimate from transmission spectra), and $\alpha=0$ for $\mathrm{SiO}_{2}$ (idealization of a non-absorbing substrate). Lacking data on $\mathrm{SiO}_{x}$ for $c_{p}$ and $\kappa$ the parameter values for fused silica are used which represents a further simplification within the used model. The laser pulse is modeled as a $\tau=20 \mathrm{~ns}$ Gaussian pulse.

First, we consider ablation at $248 \mathrm{~nm}$ of a $20 \mathrm{~nm}$ thick $\mathrm{SiO}_{x}$ film. In this case, the film thickness is less than the 
absorption length $\alpha^{-1}$ of around $40 \mathrm{~nm}$ and significantly less than the thermal diffusion length $\sqrt{\kappa \tau /\left(\rho c_{p}\right)}$ of around $100 \mathrm{~nm}$. Not surprisingly, heat equilibration is so rapid that the temporal dependence of the temperature is nearly identical at all depths $z$ within the film which means that the same maximum temperature is reached throughout the film. This result is in agreement with the experimental observation that no discernible difference is observed in the threshold fluence for FSA and RSA. Thus, the model supports the hypothesis that ablation of $\mathrm{SiO}_{x}$ films in this thickness range comprises melting and evaporation of the whole film regardless of ablation mode. For thicker films and FSA, the numerical results do not suggest an influence of film thickness on the ablation process. Temperatures calculated for a $200 \mathrm{~nm}$ and a $400 \mathrm{~nm}$ film are similar. Thus, our simple thermal model would suggest that FSA of these films is not different from bulk ablation which is in fair agreement with the experimental results shown in Fig. 2. This behavior is different from that observed for metal films of a similar thickness where the threshold fluence for ablation is thickness-dependent [21]. Because of the higher thermal conductivity heat equilibrates more rapidly in a metal film and is effectively confined there if the supporting substrate has a lower thermal conductivity as is the case for fused silica. In $\mathrm{SiO}_{x}$ heat equilibration is slower and, thus, large thermal gradients can build up during irradiation by the laser pulse. The maximum temperature reached during irradiation is highest at the film surface and drops with increasing depth into the film, falling below the threshold required for ablation at some distance from the surface.

For RSA the situation is a bit different. In this ablation geometry, the laser pulse heats a small zone near the filmsubstrate interface. From this zone heat can flow away into the substrate and into the film The heat flux in the film terminates, however, at the front film surface which serves as an isolating barrier. For thin films this can lead to an accumulation of heat so that a higher temperature is reached at the film-substrate interface than would be the case in thicker films. We investigate this effect with respect to a simple model view of the ablation process. From our experimental results we hypothesize that $\mathrm{RSA}$ of thick $\mathrm{SiO}_{x}$ films does not involve melting and evaporation of the whole film but is better characterized as a process where only a thin layer near the substrate evaporates resulting in delamination of the film. Then, we calculate the laser fluence required to reach a threshold temperature at the film-substrate interface in dependence of the film thickness. Lacking data for $\mathrm{SiO}_{x}$, we arbitrarily take this threshold temperature as the evaporation temperature of $\mathrm{SiO}$, given as $1880^{\circ} \mathrm{C}$ in [22] (the melting temperature according to that reference is $>1700^{\circ} \mathrm{C}$ ). The calculated threshold fluence using this simple model does show a dependence on film thickness, being smaller for thinner films and reaching a constant value for thick films.
However, compared to the experimental values the calculated threshold fluences come out a factor of 2 to 3 too low and stagnate at too low film thicknesses. This indicates that a detailed analysis has to take additional effects into account that are non-thermal. Mechanical effects might be important in this respect.

\section{Conclusion}

Our results demonstrate that single-pulse $\mathrm{RSA}$ of $\mathrm{SiO}_{x}$ thin films on fused silica is a practical method to produce $\mathrm{SiO}_{x}$ on- $\mathrm{SiO}_{2}$ or (by oxidation) all- $\mathrm{SiO}_{2}$ microstructures by excimer laser micromachining at $193 \mathrm{~nm}$ and $248 \mathrm{~nm}$. For a given wavelength and film thickness a fluence range exists where RSA results in removal of the $\mathrm{SiO}_{x}$ film without damage to the supporting substrate. Within that range, obtained etch depths are equal to within about 10 percent, the remaining slight fluence dependence being an interesting open question. Above that range, significant etching of the substrate occurs at $193 \mathrm{~nm}$, which might itself be a useful effect for micromachining of fused silica. For a given wavelength the threshold fluence required for $\mathrm{RSA}$ of $\mathrm{SiO}_{x}$ thin films shows a clear dependence on film thickness. Analysis using a simple thermal model hints at heat diffusion playing a part in this effect, but a more detailed description is necessary, which possibly needs to take into account mechanical effects within the film-substrate system.

Acknowledgement Financial support by the German Federal Ministry of Economics and Technology (grant no. 16IN0505) is gratefully acknowledged.

\section{References}

1. J. Ihlemann, M. Schulz-Ruhtenberg, T. Fricke-Begemann, J. Phys. Conf. Ser. 59, 206 (2007)

2. P.R. Herman, R.S. Marjoribanks, A. Oettl, K. Chen, I. Konovalov, S. Ness, Appl. Surf. Sci. 154-155, 577-586 (2000)

3. J. Ihlemann, S. Müller, S. Puschmann, D. Schäfer, M. Wei, J. Li, P.R. Herman, Appl. Phys. A 76, 751 (2003)

4. A.A. Tseng, Phys. Stat. Solidi A 204, 709 (2007)

5. J. Dou, J. Li, P.R. Herman, J.S. Aitchison, T. Fricke-Begemann, J. Ihlemann, G. Marowsky, Appl. Phys. A 91, 591 (2008)

6. J. Wang, J. Niino, A. Yabe, Appl. Phys. A 68, 111-113 (1999)

7. K. Zimmer, R. Böhme, B. Rauschenbach, J. Laser Micro/Nanoeng. 1, 292 (2006)

8. Y. Kawaguchi, H. Niino, T. Sato, A. Narazaki, R. Kurosaki, J. Phys. Conf. Ser. 59, 380 (2007)

9. J. Zhang, K. Sugioka, K. Midorikawa, Opt. Lett. 23, 1486-1488 (1998)

10. K. Zimmer, R. Böhme, B. Rauschenbach, Appl. Phys. A 79, 18831885 (2004)

11. B. Hopp, Cs. Vass, T. Smausz, Appl. Surf. Sci. 253, 7922 (2007)

12. T. Smausz, T. Csizmadia, N. Kresz, Cs. Vass, Zs. Márton, B. Hopp, Appl. Surf. Sci. 254, 1091 (2007)

13. B. Hopp, T. Smausz, T. Csizmadia, J. Budai, A. Oszkó, G. Szabó, J. Phys. D 41, 175501 (2008) 
14. M. Schulz-Ruhtenberg, J. Ihlemann, J. Heber, Appl. Surf. Sci. 248, 190 (2005)

15. J.-H. Klein-Wiele, J. Békési, P. Simon, J. Ihlemann, JLMN—J. Laser Micro/Nanoeng. 1, 211 (2006)

16. J. Ihlemann, R. Weichenhain-Schriever, J. Laser Micro/Nanoeng. 4, $100(2009)$

17. G. Hass, C.D. Salzberg, J. Opt. Soc. Am. 44, 181-187 (1954)

18. J. Ihlemann, Appl. Phys. A 93, 75 (2008)

19. German Wikipedia on silicon monoxide: http://de.wikipedia.org/ wiki/Siliciummonoxid
20. Heraeus Quarzschmelze, Transparent and Opaque Fused Silica, Document Q-A 1/112.2

21. E. Matthias, M. Reichling, J. Siegel, O.W. Käding, S. Petzold, H. Skurk, P. Bizenberger, E. Neske, Appl. Phys. A 58, 129-136 (1994)

22. R.C. Weast, M.J. Astle, CRC Handbook of Chemistry and Physics, 64th edn. (CRC Press, Boca Raton, 1983) 